\section{Gain-Phase Margin Analysis of Dynamic Fuzzy Control Systems}

Jau-Woei Perng, Bing-Fei Wu, Hung-I Chin, and Tsu-Tian Lee

\begin{abstract}
In this paper, we apply some effective methods, including the gain-phase margin tester, describing function and parameter plane, to predict the limit cycles of dynamic fuzzy control systems with adjustable parameters. Both continuous-time and sampled-data fuzzy control systems are considered. In general, fuzzy control systems are nonlinear. By use of the classical method of describing functions, the dynamic fuzzy controller may be linearized first. According to the stability equations and parameter plane methods, the stability of the equivalent linearized system with adjustable parameters is then analyzed. In addition, a simple approach is also proposed to determine the gain margin and phase margin which limit cycles can occur for robustness. Two examples of continuous-time fuzzy control systems with and without nonlinearity are presented to demonstrate the design procedure. Finally, this approach is also extended to a sampled-data fuzzy control system.
\end{abstract}

Index Terms-Fuzzy control, limit cycle, gain-phase margin, describing function, parameter plane.

\section{INTRODUCTION}

The describing function method [18] has been widely used to analyze the nonlinearcontrol systems in the frequency domain. In the recent years, limit cycle analysis of fuzzy control systems has been considered due to the describing function method. The transient and steady-state analysis of a three-term fuzzy controller has been addressed by Abdelnour et al. [1], [2]. According to the Nyquist stability criterion, the stability of fuzzy control systems has been analyzed in [10], [13], and [15]. In [14], the analytic describing function of fuzzy logic control was derived and used to predict the existence of limit cycles. On the other hand, the robust analysis of uncertain parameters in linear control system is often dealt with parameter plane method or parameter space method [3], [4], [11], [16]. Besides, this approach is also applied to the analysis of nonlinear systems in [12] and [17]. In the frequency domain, gain margin and phase margin are two important specifications for control design. Chang and Han [5] have presented a simple method to analyze the gain-phase margins of control system with adjustable parameters. After then, they have also extended the method to the nuclear reactor control system with multiple transport lags [6]. Furthermore, the gain-phase margin analysis of nonlinear control systems has been addressed in [7]-[9].

In this paper, based on the results in [14], we apply the parameter plane method to predict the limit cycles of dynamic fuzzy control systems with adjustable parameters firstly. For the robust design, a novel approach is also proposed to figure out the gain margin and phase margin of dynamic fuzzy control systems when limit cycles can occur. The information about the stability analysis of dynamic fuzzy control systems could be acquired by our approach.

The paper is organized as follows. Section II outlines the describing function analysis of dynamic fuzzy control system. Section III presents the gain-phase margin analysis of nonlinear control systems. In Section IV, three examples are referred to demonstrate the design procedure. Finally, some conclusions are made in Section V.

Manuscript received September 9, 2003; revised January 28, 2004. This work was supported by the R.O.C. Ministry of Education under Grant 91X104EX-91-E-FA06-4-4. This paper was recommended by Associate Editor J. Wang.

The authors are with the Department of Electrical and Control Engineering, National Chiao Tung University, Hsinchu, 300 Taiwan, R.O.C. (e-mail: jwperng@cn.nctu.edu.tw; bwu@cc.nctu.edu.tw; chinhi.ece85g@nctu.edu.tw; ttlee@cn.nctu.edu.tw).

Digital Object Identifier 10.1109/TSMCB.2004.831772

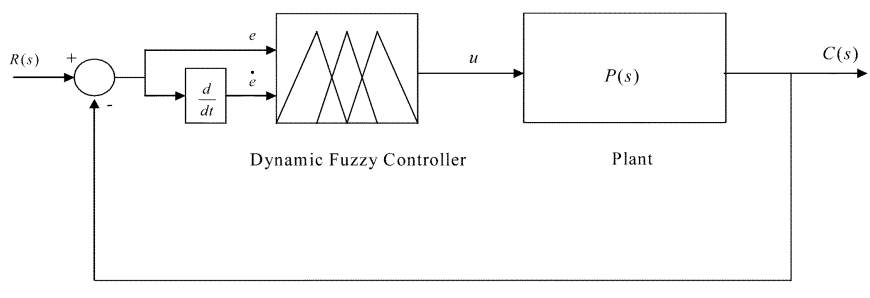

Fig. 1. Dynamic fuzzy control system.

\section{Describing Function of DynAmic FuZZy Control Systems}

In this section, the describing function analysis of dynamic fuzzy controller is presented. The block diagram of a general dynamic fuzzy control system is shown in Fig. 1 and the premise triangular membership functions of dynamic fuzzy controller are depicted in Fig. 2. If the error signal $e(t)$ shown in Fig. 1 is selected as the reference input signal $x(t)$, then the following IF-THEN fuzzy rule base is adopted:

$$
R_{i, j}: \text { If } x \text { is } \bar{M}_{i} \text { and } \dot{x} \text { is } \bar{N}_{j} \text {, then } u \text { is } u_{i, j},
$$

where $\bar{M}_{i}$ and $\bar{N}_{j}$ are fuzzy variables

$$
\begin{aligned}
& \bar{M}_{i}(x)= \begin{cases}\frac{x-\Phi_{i-1}}{\Phi_{i}-\Phi_{i-1}}, & \text { if } \Phi_{i-1} \leq x<\Phi_{i} \\
\frac{x-\Phi_{i+1}}{\Phi_{i}-\Phi_{i+1}}, & \text { if } \Phi_{i} \leq x<\Phi_{i+1} \\
0, & \text { otherwise }\end{cases} \\
& \bar{N}_{j}(\dot{x})= \begin{cases}\frac{\dot{x}-\Psi_{j-1}}{\Psi_{j}-\Psi_{j-1},} & \text { if } \Psi_{j-1} \leq \dot{x}<\Psi_{j} \\
\frac{\dot{x}-\Psi_{j+1}}{\Psi_{j}-\Psi_{j+1},} & \text { if } \Psi_{j} \leq \dot{x}<\Psi_{j+1} \\
0 & \text { otherwise, }\end{cases}
\end{aligned}
$$

and $\Phi_{-i}=-\Phi_{i}, \Psi_{-j}=-\Psi_{j}$.

If the fuzzifier is a singleton, the inference engine is a product inference and the defuzzifier is the center average, then the dynamic fuzzy control can be formulated by the following equation [14]:

$$
\begin{aligned}
u=f(x, \dot{x}) & =\sum_{i} \sum_{j}\left\{\frac{\bar{M}_{i}(x) \bar{N}_{j}(\dot{x})}{\sum_{s=-q}^{q} \sum_{r=-p}^{p} \bar{M}_{r}(x) \bar{N}_{s}(\dot{x})}\right\} u_{i, j} \\
& =\sum_{i} \sum_{j} \Omega_{i, j}(x, \dot{x}) u_{i, j}
\end{aligned}
$$

where $\Omega_{i, j}(x, \dot{x})$ is fuzzy basis function, which can be defined as [19], [20]

$$
\Omega_{i, j}(x, \dot{x})=\frac{\bar{M}_{i}(x) \bar{N}_{j}(\dot{x})}{\sum_{s=-q}^{q} \sum_{r=-p}^{p} \bar{M}_{r}(x) \bar{N}_{s}(\dot{x})} .
$$

Definition 1 [14]: Suppose that the signals input to membership functions of the dynamic fuzzy controller are $x(t)=A \sin \omega t$ and $\dot{x}(t)=\omega A \cos \omega t$, respectively, and $\Phi_{n} \leq A<\Phi_{n+1}$ and $\Psi_{m} \leq$ $\omega A<\Psi_{m+1}$. Then $\left\{\alpha_{i}\right\},\left\{\beta_{j}\right\}$, and $\left\{\gamma_{k}\right\}$ are defined as follows:

$$
\begin{aligned}
& \alpha_{i} \equiv \sin ^{-1} \frac{\Phi_{i}}{A} \quad\left(i=0, \ldots, n, 0 \leq \alpha_{i}<\frac{\pi}{2}\right) \\
& \alpha_{i} \equiv \pi-\alpha_{2 n+1-i} \quad\left(i=n+1, \ldots, 2 n+1, \frac{\pi}{2} \leq \alpha_{i}<\pi\right) \\
& \beta_{0} \equiv 0, \quad \beta_{j} \equiv \cos ^{-1} \frac{\Psi_{m-j+1}}{\omega A} \\
& \quad\left(j=1, \ldots, 2 m+1,0<\beta_{j}<\pi\right) \text { and } \beta_{2 m+2}=\pi .
\end{aligned}
$$

$\left\{\gamma_{k}\right\}(k=0, \ldots, h)$ is defined as the sorted values of $\left\{\alpha_{i}\right\}$ and $\left\{\beta_{j}\right\}$ in the ascending order, where $h+1$ is the number of $\left\{\gamma_{k}\right\}$. 


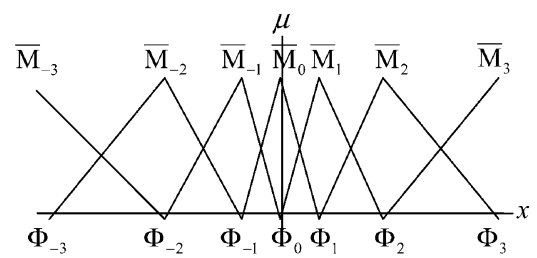

Fig. 2. Premise membership functions of dynamic fuzzy controller.

Lemma 1 [14]: For $\Phi_{k} \leq x<\Phi_{k+1}$ and $\Psi_{l} \leq \dot{x}<\Psi_{l+1}$, the dynamic fuzzy control is formulated as the following equations

$$
\begin{aligned}
u(x, \dot{x}) & =\sum_{j=-q}^{q} \sum_{i=-p}^{p} \Omega_{i, j}(x, \dot{x}) u_{i, j} \\
& =a_{k, l} \frac{x}{\Delta \Phi_{k}} \frac{\dot{x}}{\Delta \Psi_{l}}+b_{k, l} \frac{x}{\Delta \Phi_{k}}+c_{k, l} \frac{\dot{x}}{\Delta \Psi_{l}}+d_{k, l}
\end{aligned}
$$

where

$$
\begin{aligned}
& \Delta \Phi_{k}=\Phi_{k+1}-\Phi_{k}, \quad \Delta \Psi_{l}=\Psi_{l+1}-\Psi_{l} \\
& a_{k, l} \equiv u_{k, l}-u_{k, l+1}-u_{k+1, l}+u_{k+1, l+1} \\
& b_{k, l} \equiv \frac{1}{\Delta \Psi_{l}}\left(\Psi_{l+1}\left(u_{k+1, l}-u_{k, l}\right) \Psi_{l}\left(u_{k, l+1}-u_{k+1, l+1}\right)\right) \\
& c_{k, l} \equiv \frac{1}{\Delta \Phi_{k}}\left(\Phi_{k+1}\left(u_{k, l+1}-u_{k, l}\right)+\Phi_{k}\left(u_{k+1, l}-u_{k+1, l+1}\right)\right) \\
& d_{k, l} \equiv \\
& \frac{1}{\Delta \Phi_{k}} \frac{1}{\Delta \Psi_{l}}\left(\Phi_{k+1} \Psi_{l+1} u_{k, l}-\Phi_{k+1} \Psi_{l} u_{k, l+1}\right. \\
&\left.-\Phi_{k} \Psi_{l+1} u_{k+1, l}+\Phi_{k} \Psi_{l} u_{k+1, l+1}\right) .
\end{aligned}
$$

Theorem 1 [14]: The describing function of dynamic fuzzy controller is expressed by the following equation:

$$
N_{1} \equiv N_{1}(A, \omega)=\frac{1}{A}\left(b_{1}+j a_{1}\right)
$$

where

$$
\begin{aligned}
a_{1}= & \frac{2}{\pi} \sum_{i=0}^{h-1}\left\{-a_{k i, l i} \frac{A^{2} \omega}{3 \Delta \Phi_{k i} \Delta \Psi_{l i}}\left(\cos ^{3} \gamma_{i+1}-\cos ^{3} \gamma_{i}\right)\right. \\
& -b_{k i, l i} \frac{A}{4 \Delta \Phi_{k i}}\left(\cos 2 \gamma_{i+1}-\cos 2 \gamma_{i}\right) \\
& +c_{k i, l i} \frac{A \omega}{2 \Delta \Psi_{l i}}\left(\left(\gamma_{i+1}-\gamma_{i}\right)\right. \\
& \left.+\left(\sin \gamma_{i+1} \cos \gamma_{i+1}-\sin \gamma_{i} \cos \gamma_{i}\right)\right) \\
& \left.+d_{k i, l i}\left(\sin \gamma_{i+1}-\sin \gamma_{i}\right)\right\} \\
b_{1}= & \frac{2}{\pi} \sum_{i=0}^{h-1}\left\{a_{k i, l i} \frac{A^{2} \omega}{3 \Delta \Phi_{k i} \Delta \Psi_{l i}}\left(\sin ^{3} \gamma_{i+1}-\sin ^{3} \gamma_{i}\right)\right. \\
& +b_{k i, l i} \frac{A}{2 \Delta \Phi_{k i}}\left(\left(\gamma_{i+1}-\gamma_{i}\right)-\sin \gamma_{i+1} \cos \gamma_{i+1}\right. \\
& \left.+\sin \gamma_{i} \cos \gamma_{i}\right)-c_{k i, l i} \frac{A \omega}{4 \Delta \Psi_{l i}}\left(\cos 2 \gamma_{i+1}-\cos 2 \gamma_{i}\right) \\
& \left.-d_{k i, l i}\left(\cos \gamma_{i+1}-\cos \gamma_{i}\right)\right\}
\end{aligned}
$$

where $\left\{\gamma_{i}\right\}$ and $h$ are given in Definition $1 ; k i$ and $l i$ are defined to satisfy $\Phi_{k i} \leq A \sin \gamma<\Phi_{k i+1}$ and $\Psi_{l i} \leq \omega A \cos \gamma<\Psi_{l i+1}$, respectively, for $\gamma_{i} \leq \gamma<\gamma_{i+1} ; a_{k i, l i}, b_{k i, l i}, c_{k i, l i}$, and $d_{k i, l i}$ are given in Lemma 1.

Remark 1: From Theorem 1, it can be noted that the zero term $a_{0}$ of describing function of dynamic fuzzy controller is disappeared since the consequent parts meet the odd condition.

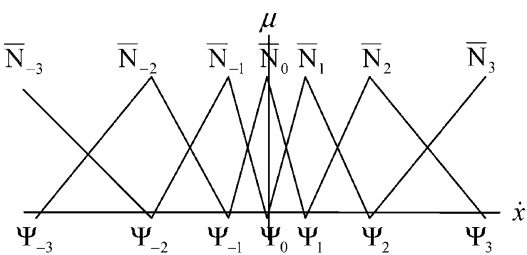

\section{Gain-Phase Margin AnAlysis OF NONLINEAR CONTROL SYSTEMS}

A systematic procedure, including the parameter plane method and gain-phase margin tester method, is proposed to predict limit cycles of nonlinear control system with adjustable parameters. In Fig. 3, a general linearized system with multiple nonlinear elements is considered, where $G\left(s, N_{1 R}, N_{1 I}, \ldots, N_{m R}, N_{m I}\right)$ is the open-loop transfer function. $N_{1 R}, \ldots, N_{m R}$ and $N_{1 I}, \ldots, N_{m I}$ are real parts and imaginary parts of the describing function $\left(N_{i}\right)$ of $n_{1}, n_{2}, \ldots, n_{m}$, respectively, which can be expressed in the following equation [7]:

$$
N_{i}(A, \omega)=N_{i R}(A, \omega)+j N_{i I}(A, \omega), \quad i=1, \ldots, m
$$

where $A$ and $\omega$ are the amplitude and frequency of sinusoidal input to one of the nonlinearities. Besides, a gain-phase margin tester $\left(K e^{-j \theta}\right)$ is also inserted in the forward part of open-loop transfer function.

After some simple manipulations, the characteristic equation of this equivalent linear system can be expressed as

$$
\begin{aligned}
1+ & K e^{-j \theta} G\left(s, N_{1 R}, N_{1 I}, \ldots, N_{m R}, \ldots, N_{m I}\right) \\
& =1+K e^{-j \theta} \frac{N\left(s, N_{1 R}, N_{1 I}, \ldots, N_{m R}, \ldots, N_{m I}\right)}{D\left(s, N_{1 R}, N_{1 I}, \ldots, N_{m R}, \ldots, N_{m I}\right)}=0
\end{aligned}
$$

which is equivalent to

$$
\begin{aligned}
f(s) \triangleq & D\left(s, N_{1 R}, N_{1 I}, \ldots, N_{m R}, \ldots, N_{m I}\right) \\
& +K e^{-j \theta} N\left(s, N_{1 R}, N_{1 I}, \ldots, N_{m R}, \ldots, N_{m I}\right)=0 .
\end{aligned}
$$

Let $s=j \omega$; one has

$$
f(j \omega)=f(\alpha, \beta, \gamma, \ldots, K, \theta, j \omega)=0
$$

where $\alpha, \beta, \gamma, \ldots$ are variables which consist of the items $\left(N_{i R}, N_{i I}\right)$ of describing functions and/or adjustable parameters of the linear portion of the system. Notice that the designer can define these variables arbitrarily in order to analyze the effect of system parameters. When only two decoupled parameters $\alpha$ and $\beta$ are chosen to concern, (8) is arranged as the following equation:

$$
f(j \omega)=f(\alpha, \beta, \gamma, \ldots, K, \theta, j \omega)=X \cdot \alpha+Y \cdot \beta+Z=0,
$$

where $X, Y$, and $Z$ are functions of $\gamma, \ldots, K, \theta$, and $j \omega$. Let (9) be partitioned into two stability equations with real part $\left(f_{R}\right)$ and imaginary part $\left(f_{I}\right)$ and written in the following [12]:

$$
f_{R}(\alpha, \beta, \gamma, \ldots, K, \theta, \omega)=X_{1} \cdot \alpha+Y_{1} \cdot \beta+Z_{1}=0
$$

and

$$
f_{I}(\alpha, \beta, \gamma, \ldots, K, \theta, \omega)=X_{2} \cdot \alpha+Y_{2} \cdot \beta+Z_{2}=0
$$

where $X_{1}, Y_{1}, Z_{1}$, and $X_{2}, Y_{2}, Z_{2}$ are real and imaginary parts of $X, Y$, and $Z$. Therefore, $\alpha$ and $\beta$ are solved from (10) and (11), one has

$$
\alpha=\frac{Y_{1} \cdot Z_{2}-Y_{2} \cdot Z_{1}}{\Delta}
$$




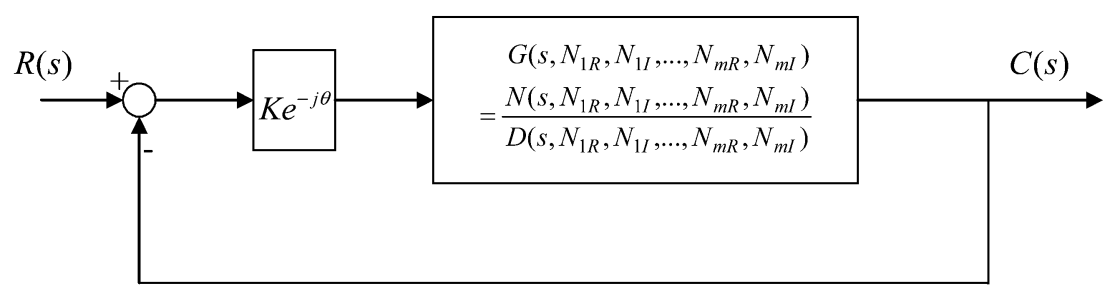

Fig. 3. A general linearized system including gain-phase margin tester.

and

$$
\beta=\frac{Z_{1} \cdot X_{2}-Z_{2} \cdot X_{1}}{\Delta}
$$

where $\Delta=X_{1} \cdot Y_{2}-X_{2} \cdot Y_{1}$.

On the other hand, let $\theta=0^{\circ}$; (8) is rearranged as follows:

$$
f(j \omega)=f(\alpha, \beta, \gamma, \ldots, K, j \omega)=E \cdot K+F=0 .
$$

Partitioning (14) into real and imaginary parts yields

$$
f_{R}(\alpha, \beta, \gamma, \ldots, K, \omega)=E_{1} \cdot K+F_{1}=0
$$

and

$$
f_{I}(\alpha, \beta, \gamma, \ldots, K, \omega)=E_{2} \cdot K+F_{2}=0
$$

where $E_{1}, E_{2}, F_{1}$, and $F_{2}$ are functions of $\alpha, \beta, \gamma, \ldots$, and $\omega$. Thus, $K$ can be obtained from (15) and (16), which yield

$$
K=\frac{-F_{1}}{E_{1}} \triangleq K^{\prime}
$$

and

$$
K=\frac{-F_{2}}{E_{2}} \triangleq K^{\prime \prime}
$$

If $K^{\prime}=K^{\prime \prime}=K_{i}$ for $A=A_{i}$, the values of $A_{i}$ and $K_{i}$ related to $\omega_{i}$ can be found by varying $A$ from 0 to $\infty$. For many values of $\omega$, a set (GM) of desired values of $A$ and $K$ can be obtained. Alternatively, let $k=0 \mathrm{~dB} ;(8)$ is rearranged as follows:

$$
f(j \omega)=f(\alpha, \beta, \gamma, \ldots, \theta, j \omega)=U \cdot \cos \theta+V \cdot \sin \theta+W=0 .
$$

Also, partitioning (19) into real and imaginary parts yields

$$
f_{R}(\alpha, \beta, \gamma, \ldots, \theta, \omega)=U_{1} \cdot \cos \theta+V_{1} \cdot \sin \theta+W_{1}=0
$$

and

$$
f_{I}(\alpha, \beta, \gamma, \ldots, \theta, \omega)=U_{2} \cdot \cos \theta+V_{2} \cdot \sin \theta+W_{2}=0
$$

where $U_{1}, V_{1}, W_{1}, U_{2}, V_{2}$, and $W_{2}$ are functions of $\alpha, \beta, \gamma, \ldots$, and $\omega$. Hence, $\theta$ can be obtained from (20) and (21), which yield

$$
\theta=\cos ^{-1}\left(\frac{V_{1} \cdot W_{2}-V_{2} \cdot W_{1}}{U_{1} \cdot V_{2}-U_{2} \cdot V_{1}}\right) \triangleq \theta^{\prime}
$$

and

$$
\theta=\sin ^{-1}\left(\frac{U_{1} \cdot W_{2}-U_{2} \cdot W_{1}}{U_{1} \cdot V_{2}-U_{2} \cdot V_{1}}\right) \triangleq \theta^{\prime \prime}
$$

If $\theta^{\prime}=\theta^{\prime \prime}=\theta_{i}$ for $A=A_{i}, A_{i}$, and $\theta_{i}$ related to $\omega_{i}$ can be found by varying $A$ from 0 to $\infty$. For many values of $\omega$, a set (PM) of desired values for $A$ and $\theta$ can be obtained.

Based on the above analysis, if any two adjustable parameters operating in the asymptotically stable region are considered for stability margin analysis, then $\mathrm{GM}_{\mathrm{min}}$ and $\mathrm{PM}_{\min }$ defined as the minimum values of GM and PM represent the minimum amounts by which the loop gain and phase shift should be increased to produce a limit cycle solution.
Finally, the similar procedure applied to the stability analysis of nonlinear sampled-data control systems can be listed as follows.

Step 1) Augment the characteristic equation as (7) of a nonlinear sampled-data system.

Step 2) Let $z=e^{j \omega T}$, where $T$ is the sampling period and evaluate (8).

Step 3) Solve $\alpha$ and $\beta$ by using (10)-(13).

Step 4) Gain margin analysis by using (14)-(18).

Step 5) Phase margin analysis by using (19)-(23).

\section{SIMULATION EXAMPLES}

In this section, three examples of dynamic fuzzy control system are cited to verify the design procedure. The first example is a third-order system. The second example is a third-order system with a nonlinear element of saturation. The final example is a sample-data system.

\section{A. Example 1}

Consider the dynamic fuzzy control system shown in Fig. 1 and a third-order equation in the following is adopted:

$$
P(s)=\frac{1}{s^{3}+q_{1} s^{2}+q_{2} s+1} .
$$

In this simulation, the 25 rules listed in Table I and triangular membership functions shown in Fig. 4 are picked [14]. If the parameters of fuzzy rules listed in Table II are assumed and the corresponding parameters are as follows:

$$
\begin{aligned}
\bar{M}_{-2} & =\mathrm{NBE}, \quad \bar{M}_{-1}=\mathrm{NSE}, \quad \bar{M}_{0}=\mathrm{ZRE}, \quad \bar{M}_{1}=\mathrm{PSE} \\
\bar{M}_{2} & =\mathrm{PBE} \\
\bar{N}_{-2} & =\mathrm{NBDE}, \quad \bar{N}_{-1}=\mathrm{NSDE}, \quad \bar{N}_{0}=\mathrm{ZRDE}, \quad \bar{N}_{1}=\mathrm{PSDE} \\
\bar{N}_{2} & =\mathrm{PBDE} \\
\Phi_{-2} & =\text { nbe }, \quad \Phi_{-1}=\mathrm{nse}, \quad \Phi_{0}=\text { zre }, \Phi_{1}=\text { pse }, \quad \Phi_{2}=\text { pbe } \\
\Psi_{-2} & =\text { nbde } \\
\Psi_{-1} & =\text { nsde }, \quad \Psi_{0}=\text { zrde }, \quad \Psi_{1}=\text { psde }, \quad \Psi_{2}=\text { pbde } .
\end{aligned}
$$

Then the describing function $\left(N_{1}\right)$ of dynamic fuzzy controller can be expressed as (4) and the control surface is given in Fig. 5. It is noted that the output value in the original $(e, \dot{e})=(0,0)$ is 0 due to the center average method. When the gain-phase margin tester $\left(K e^{-j \theta}\right)$ is cascaded in the open-loop system, the overall open-loop transfer function can be expressed as

$$
G(s)=\frac{K e^{-j \theta} N_{1}}{s^{3}+q_{1} s^{2}+q_{2} s+1} .
$$

After some manipulations, the characteristic equation is

$$
\begin{aligned}
f(s) & =s^{3}+q_{1} s^{2}+q_{2} s+1+K e^{-j \theta} N_{1} \\
& =s^{2} q_{1}+s q_{2}+s^{3}+1+K e^{-j \theta} N_{1} \\
& =X \cdot \alpha+Y \cdot \beta+Z \\
& =0
\end{aligned}
$$


TABLE I

RULES OF FUZZY CONTROLLER

\begin{tabular}{c|c|c|c|c|c} 
c $_{\dot{e}}^{e}$ & NBE & NSE & ZRE & PSE & PBE \\
\hline PBDE & ZRU & PBU & PBU & PBU & PBU \\
\hline PSDE & NBU & ZRU & PSU & PSU & PBU \\
\hline ZRDE & NBU & NSU & ZRU & PSU & PBU \\
\hline NSDE & NBU & NSU & NSU & ZRU & PBU \\
\hline NBDE & NBU & NBU & NBU & NBU & ZRU \\
\hline
\end{tabular}
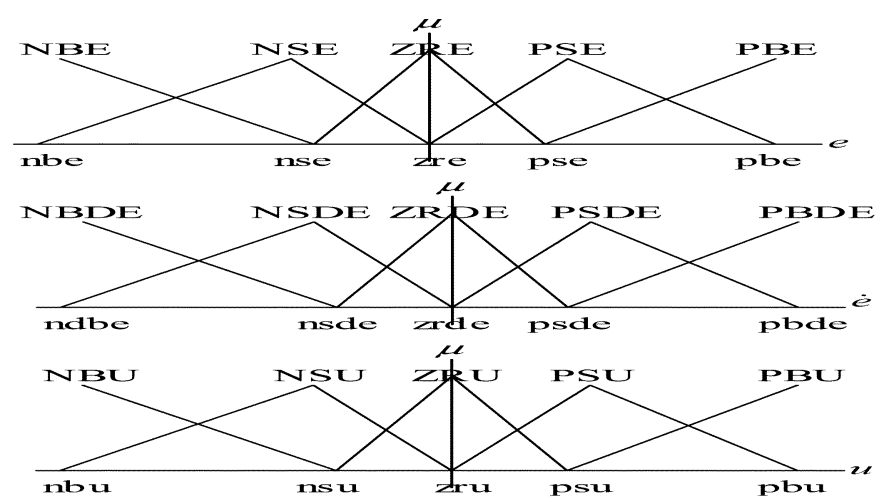

Fig. 4. Membership functions.

TABLE II

PARAMETERS OF THE RULE

\begin{tabular}{c|c|c|c|c|c}
\hline \multirow{2}{*}{$e$} & nbe & nse & zre & pse & pbe \\
\cline { 2 - 6 } & -1 & -0.02 & 0 & 0.02 & 1 \\
\hline \multirow{2}{*}{$\dot{e}$} & nbde & nsde & zrde & psde & pbde \\
\cline { 2 - 6 } & -1000 & -20 & 0 & 20 & 1000 \\
\hline \multirow{2}{*}{$u$} & nbu & nsu & zru & psu & pbu \\
\cline { 2 - 6 } & -2 & -1.4 & 0 & 1.4 & 2 \\
\hline
\end{tabular}

where $\alpha=q_{1}$ and $\beta=q_{2}$ are two adjustable parameters. Substituting $s=j \omega$ into (26), enables $\alpha$ and $\beta$ to be determined from (10)-(13). Then, the stability boundary $\left(K=0 \mathrm{~dB}, \theta=0^{\circ}\right)$ can be plotted in the $q_{1}$ versus $q_{2}$ plane with fixed amplitude $A$ (varying $\omega$ from 0 to $\infty$ ) and fixed frequency $\omega$ (varying $A$ from 0 to $\infty$ ). Fig. 6 shows some limit cycle loci. In order to test the accuracy of Fig. 6, two points $Q_{1}(4.6,4.2)$ (limit cycle region: $\left.A=0.1, \omega=2\right)$ and $Q_{2}(14,12)$ (asymptotically stable region) are selected. Fig. 7 shows the time responses of input signal $x(t)$. We can clearly find that the results in Fig. 7 are matched with Fig. 6. Due to analyzing the gain-phase margins for limit cycle prediction, the point $Q_{2}$ is chosen. First, let $\theta=0^{\circ}$. Equation (26) can be arranged as

$$
\begin{aligned}
f(s) & =N_{1} K+s^{3}+14 s^{2}+12 s+1 \\
& =E \cdot K+F \\
& =0
\end{aligned}
$$

where $E=N_{1}$ and $F=s^{3}+14 s^{2}+12 s+1$. By utilizing (15)-(18), a set of GM can be obtained and plotted in Fig. 8. On the other hand, let $K=0 \mathrm{~dB}$. Equation (26) can be also arranged as

$$
\begin{aligned}
f(s) & =N_{1} \cos \theta+(-j) N_{1} \sin \theta+s^{3}+14 s^{2}+12 s+1 \\
& =U \cdot \cos \theta+V \cdot \sin \theta+W \\
& =0 .
\end{aligned}
$$

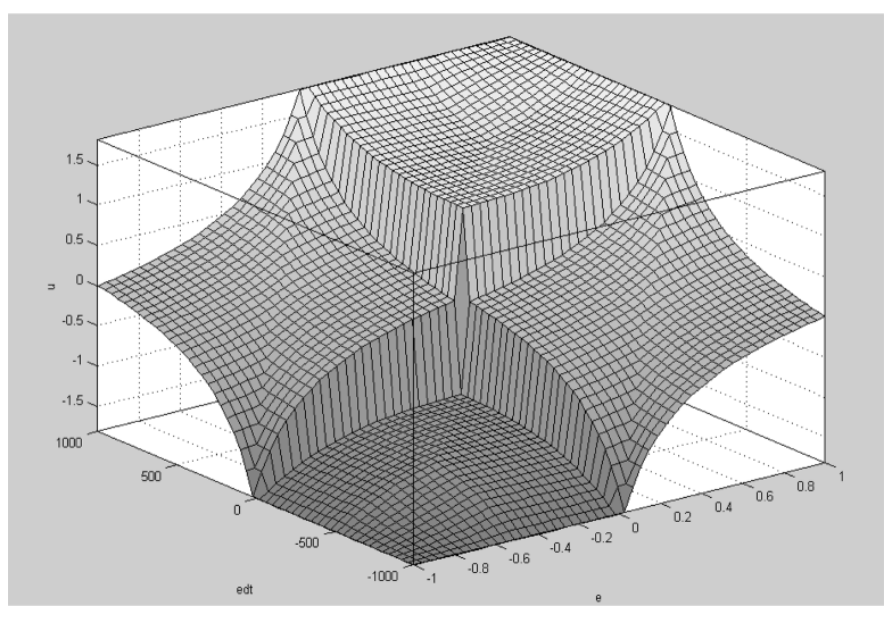

Fig. 5. Control surface.

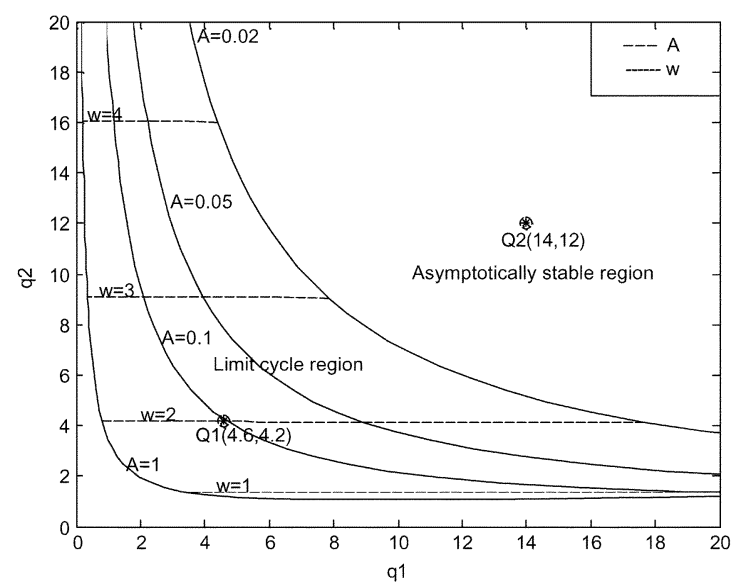

Fig. 6. Limit cycle loci in parameter plane.

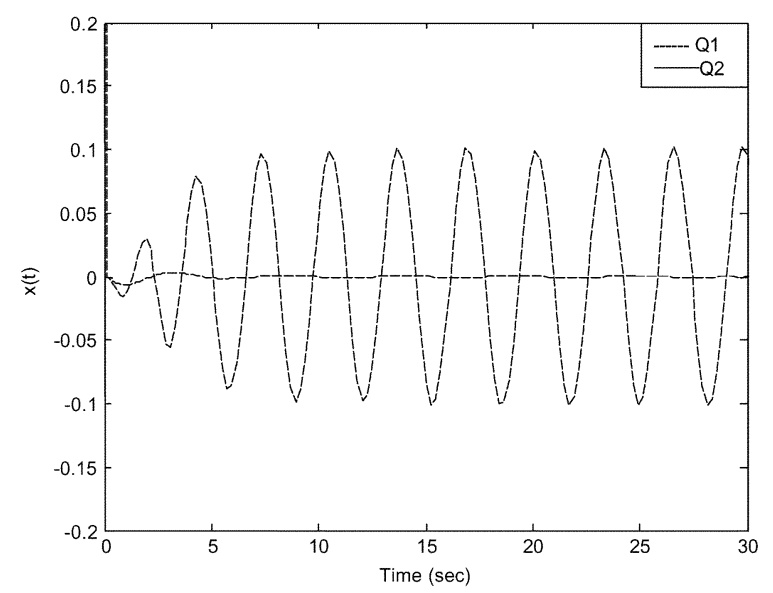

Fig. 7. Input signal $x(t)$.

where $U=N_{1}, V=(-j) N_{1}$, and $W=s^{3}+14 s^{2}+12 s+1$. By utilizing (20)-(23), a set of PM can be obtained and plotted in Fig. 8. The minimum gain margin and phase margin are $7.54 \mathrm{~dB}$ and 13.3 degrees, respectively. The relative gain-phase margins can be also checked by using Nyquist plot, which is shown in Fig. 9. In Fig. 10, the time responses of input signal $x(t)$ are depicted and the characteristics of limit cycles conform to the results in Fig. 8. 


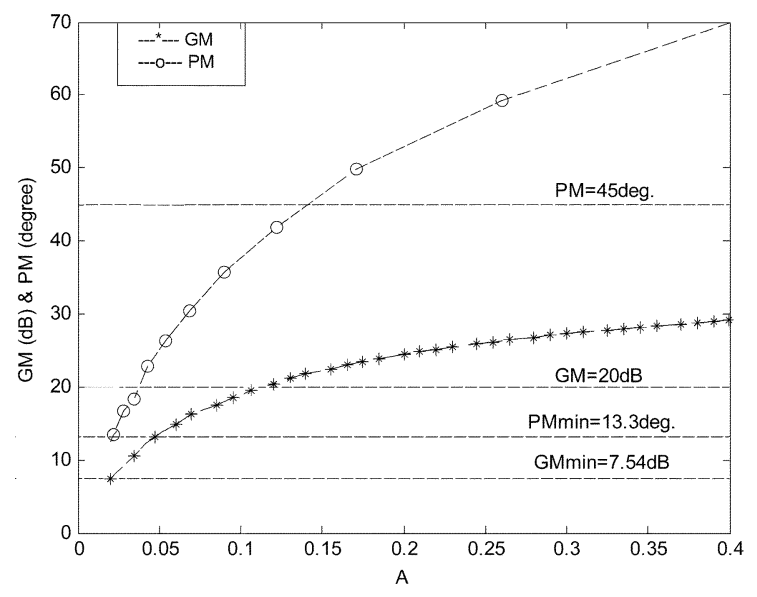

Fig. 8. Sets of GM and PM.

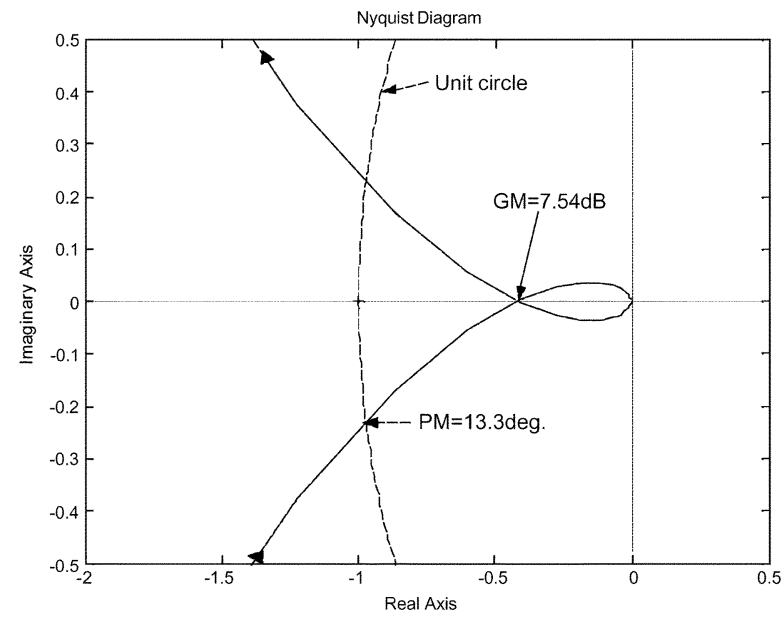

Fig. 9. Nyquist plot.

\section{B. Example 2}

Consider the dynamic fuzzy control system as the same with Example 1 and insert a nonlinear element of saturation $\left(N_{2}\right)$ into the system between the dynamic fuzzy controller and the plant. Assume that the input signals of $N_{1}$ and $N_{2}$ are $x(t)=A \sin \omega t$ and $x_{2}(t)=$ $A_{2} \sin \omega t$, respectively, the describing function of $N_{2}$ is given as [12]

$$
N_{2}=\frac{2 k}{\pi}\left(\sin ^{-1} \frac{d}{A_{2}}+\frac{d}{A_{2}}\left(1-\frac{d^{2}}{A_{2}^{2}}\right)^{\frac{1}{2}}\right)
$$

where $k=1$ and $d=0.5$.

Remark 2: If $x(t)$ is chosen as a reference input signal, $A_{2}$ can be expressed as the function of $A$, i.e., $A_{2}=A \cdot\left|N_{1}\right|$.

When the gain-phase margin tester is cascaded in the open-loop system, the overall open-loop transfer function can be expressed as

$$
G(s)=\frac{K e^{-j \theta} N_{1} N_{2}}{s^{3}+q_{1} s^{2}+q_{2} s+1} .
$$

By using a similar procedure as in Example 1, if $\alpha=q_{1}$ and $\beta=q_{2}$ are selected, some limit cycle loci are plotted in Fig. 11. As compared the results with Fig. 6, we observe that the range of limit cycle region in Fig. 11 is decreased when the saturation is added in the dynamic fuzzy control system. The gain-phase margin analysis for limit cycle prediction is fulfilled when $\theta_{2}$ is picked as the same with Example 1. Let $\theta=0^{\circ}$ and adopt the similar procedure, a set of GM can be obtained and plotted in Fig. 12. On the other hand, let $K=0 \mathrm{~dB}$, a set of $\mathrm{PM}$ can be also obtained and plotted in Fig. 12. It means that, when the

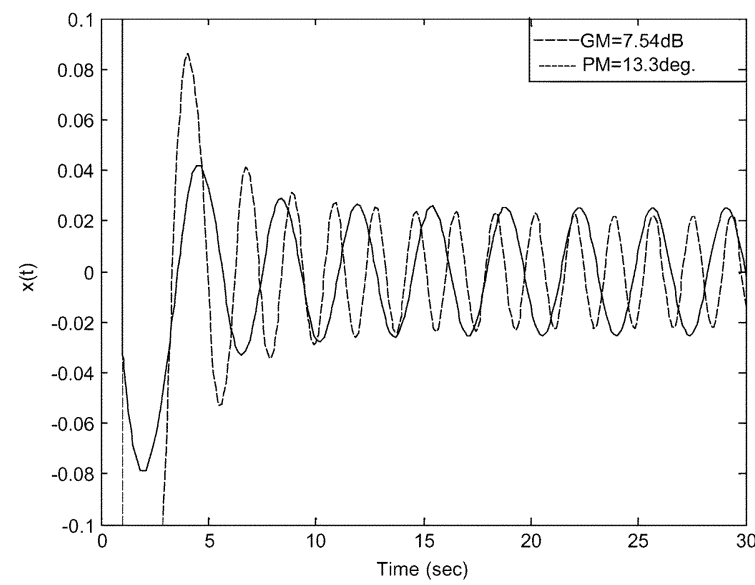

Fig. 10. Input signal $x(t)$.

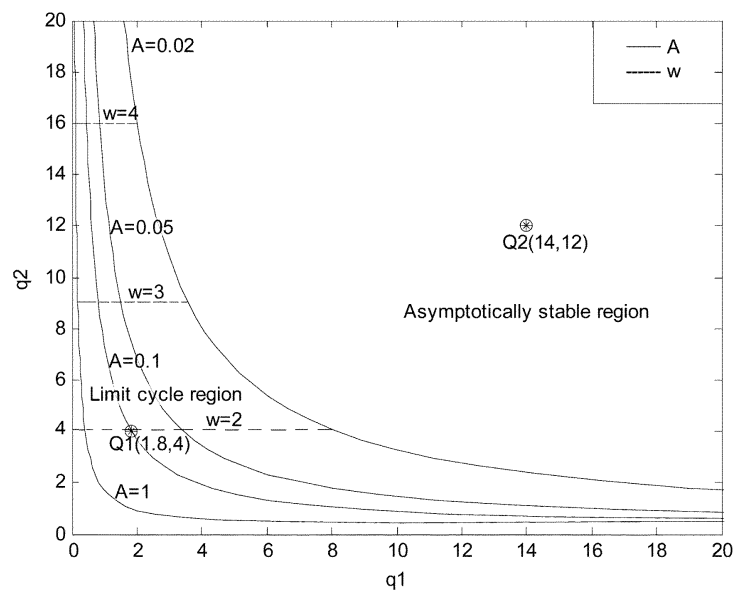

Fig. 11. Limit cycle loci in parameter plane.

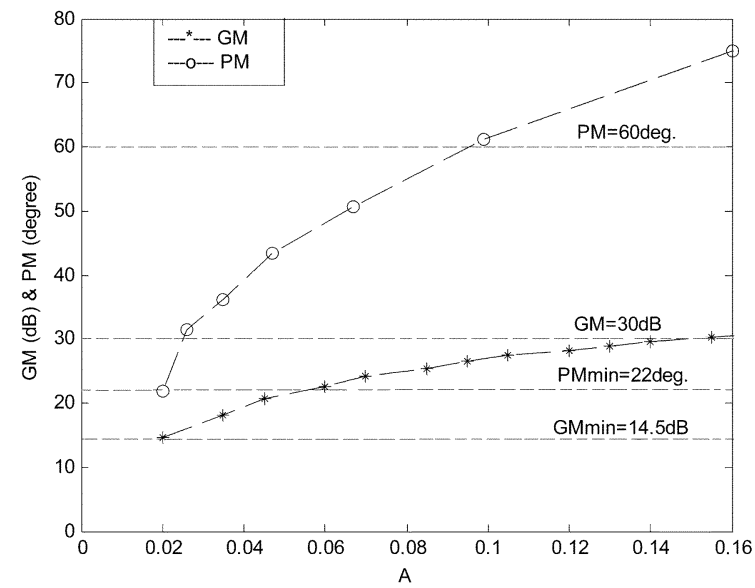

Fig. 12. Sets of GM and PM.

saturation is inserted, the dynamic fuzzy control system here is more robust than Example 1 due to prevent the occurrence of limit cycles.

\section{Example 3}

The block diagram of a sample-data dynamic fuzzy control system is depicted in Fig. 13. The transfer functions are

$$
P(s)=\frac{1.173 k(s+h)}{(s+0.01)(s+2.3)(s+0.51)}
$$




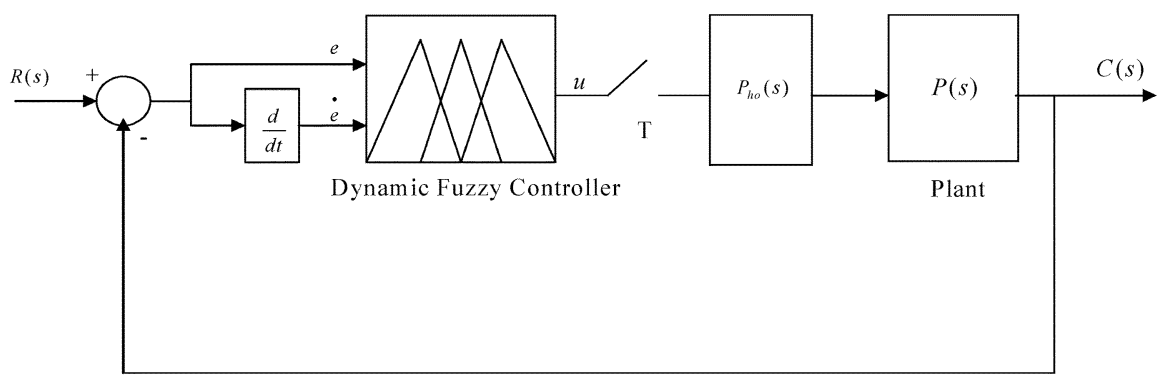

Fig. 13. Sampled-data dynamic fuzzy control system.

and

$$
P_{h o}(s)=\frac{1-e^{-T s}}{s}
$$

where the sampling period $T=1 \mathrm{~s}$.

If the describing function $\left(N_{1}\right)$ of dynamic fuzzy controller is selected as Example 1 and the gain-phase margin tester is inserted, the overall open-loop transfer function is as shown in the equation at the bottom of the page. where $Z_{\mathrm{tf}}[\cdot]$ denotes "the z-transform of [ $\left.\cdot\right]$ ".

After some simple manipulations, the characteristic equation becomes

$$
\begin{aligned}
f(z)= & z^{3}+\left(0.12 K e^{-j \theta} N_{1} k h+0.255 K e^{-j \theta} N_{1} k-1.68\right) z^{2} \\
& +\left(0.215 K e^{-j \theta} N_{1} k h-0.14 K e^{-j \theta} N_{1} k+0.746\right) z \\
& +\left(0.027 K e^{-j \theta} N_{1} k h-0.1 K e^{-j \theta} N_{1} k-0.059\right) \\
= & \left(0.12 K e^{-j \theta} N_{1} z^{2}+0.215 K e^{-j \theta} N_{1} z+0.027 K e^{-j \theta} N_{1}\right) k h \\
& +\left(0.255 K e^{-j \theta} N_{1} z^{2}-0.14 K e^{-j \theta} N_{1} z-0.1 K e^{-j \theta} N_{1}\right) k \\
& +\left(z^{3}-1.68 z^{2}+0.746 z-0.059\right) \\
= & X \cdot \alpha+Y \cdot \beta+Z=0
\end{aligned}
$$

where $\alpha=k h$ and $\beta=k$ are two adjustable parameters. Substituting $z=e^{j \omega T}$ into (34), $\alpha$ and $\beta$ are determined from (10)-(13), which can be plotted by the boundaries with fixed amplitude $A$ (varying $\omega$ from 0 to $\infty$ ) and fixed frequency $\omega$ (varying $A$ from 0 to $\infty$ ) in the $h$ vs. $k$ plane, where $h=\alpha / \beta$. Some limit-cycle loci are revealed in Fig. 14 . The gain margin and phase margin analysis for limit cycle prediction of sampled-data dynamic fuzzy control system can be performed by use of (14)-(18), and (19)-(23), respectively. In our simulations, $Q_{1}$ is adopted and the sets of GM and PM are displayed in Fig. 15.

\section{CONCLUSION}

In this paper, the limit cycle prediction of a dynamic fuzzy control system with adjustable parameters is achieved by utilizing the useful approaches of describing function, parameter plane and gain-phase margin tester. In addition, a simple method is also proposed to figure out the gain margin and phase margin when limit cycles can occur. Some examples are illustrated to demonstrate the design procedure. Computer simulations show that the information about the characteristics of limit cycles of the dynamic fuzzy control systems could be acquired by our work.

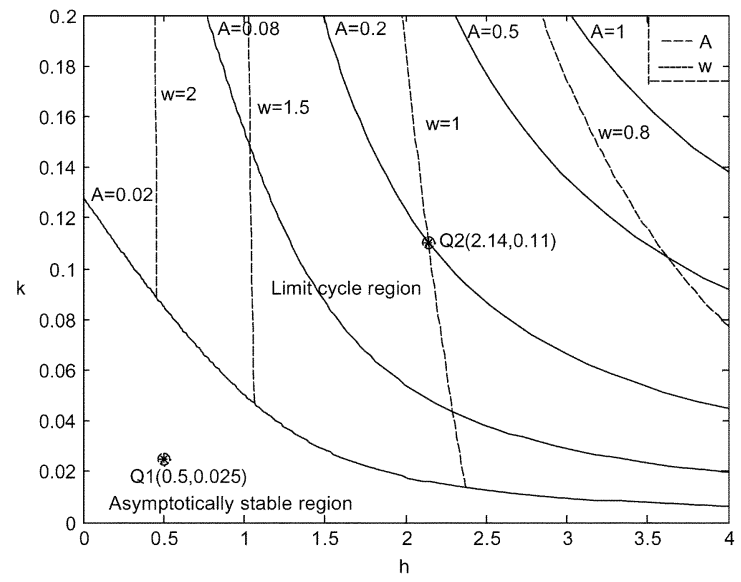

Fig. 14. Limit cycle loci in parameter plane.

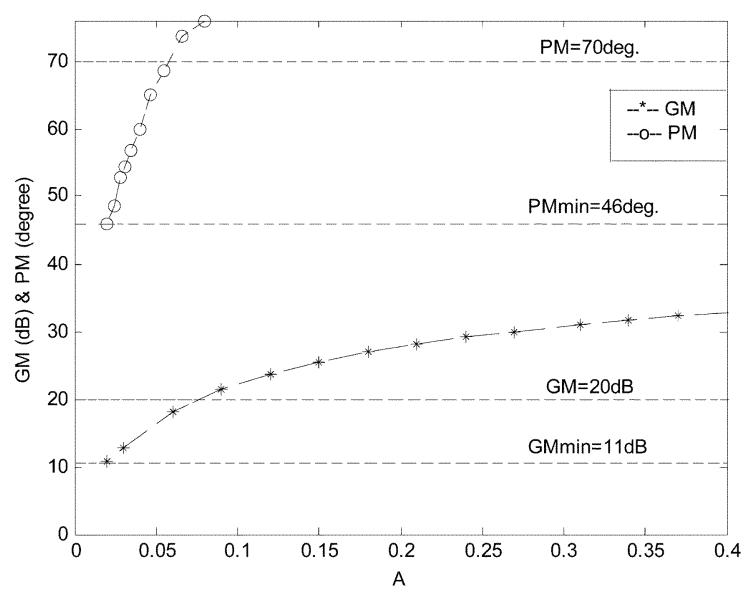

Fig. 15. Sets of GM and PM.

\section{REFERENCES}

[1] G. Abdelnour, J. Y. Cheung, C. H. Chang, and G. Tinetti, "Application of describing functions in the transient response analysis of a three-term fuzzy controller," IEEE Trans. Syst., Man, Cybern., vol. 23, pp. 603-606, Apr. 1993.

$$
\begin{aligned}
G(z) & =K e^{-j \theta} N_{1} Z_{\mathrm{tf}}\left[P_{h o}(s) P(s)\right] \\
& =K e^{-j \theta} N_{1} k \frac{(0.12 h+0.255) z^{2}+(0.215 h-0.14) z+(0.027 h-0.1)}{(z-0.98)(z-0.6)(z-0.1)}
\end{aligned}
$$


[2] — "Steady-state analysis of a three-term fuzzy controller," IEEE Trans. Syst., Man, Cybern., vol. 23, pp. 607-610, Apr. 1993.

[3] J. Ackermann, "Parameter space design of robust control systems," IEEE Trans. Automat. Contr, vol. AC-25, pp. 1058-1072, 1980.

[4] A. Cavallo, G. E. Maria, and L. Verde, "Robust control systems: A parameter space design," J. Guidance, Contr., Dynam., vol. 15, no. 5, pp. 1207-1215, 1992

[5] C. H. Chang and K. W. Han, "Gain margins and phase margins for control systems with adjustable parameters," J. Guidance, Contr., Dynam., vol. 13, no. 3, pp. 404-408, 1990.

[6] - "Gain margin and phase margin analysis of a nuclear reactor control system with multiple transport lags," IEEE Trans. Nucl. Sci., vol. 36, no. 4, pp. $1418-1425,1989$.

[7] C. H. Chang and M. K. Chang, "Analysis of gain margins and phase margins of a nonlinear reactor control system," IEEE Trans. Nucl. Sci., vol. 41, pp. 1686-1691, Aug. 1994.

[8] M. K. Chang, C. H. Chang, and K. W. Han, "Gain margins and phase margins for nonlinear control systems with adjustable parameters," in Proc. IEEE Int. Conf. Industrial Application, 1993, pp. 2123-2130.

[9] Y. C. Chang and K. W. Han, "Analysis of limit cycles for a low flying vehicle with three nonlinearities," J. Actual Prob. Aviation and Aerosp. Syst., vol. 6, no. 2, pp. 38-50, 1998.

[10] F. Gordillo, J. Aracil, and T. Alamo, "Determining limit cycles in fuzzy control systems," in Proc. IEEE Int. Conf. Fuzzy Systems, July 1997, pp. 193-198.
[11] K. W. Han and G. J. Thaler, "Control system analysis and design using a parameter space method," IEEE Trans. Automat. Contr., vol. 11, pp. 560-563, July 1966.

[12] K. W. Han, Nonlinear Control Systems-Some Practical Methods. San Diego, CA: Academic Cultural Co., 1977.

[13] X. Jun, S. Zhongke, and D. Guanzhong, "Using describing function to analyze wriggling phenomenon of fuzzy control systems," in Proc. IEEE Int. Conf. Fuzzy Systems, 1996, pp. 1198-1204.

[14] E. Kim, H. Lee, and M. Park, "Limit-cycle prediction of a fuzzy control system based on describing function method," IEEE Trans. Fuzzy Syst., vol. 8, pp. 11-21, Feb. 2000.

[15] L. Sheng and J. H. Taylor, "Fuzzy-logic controller synthesis based on sinusoidal-input describing functions \& optimization," in Proc. IEEE Int. Conf. Control Applications, 1996, pp. 219-224.

[16] D. D. Siljak, "Analysis and synthesis of feedback control systems in the parameter plane," IEEE Trans. Ind. Applicat., vol. IA-83, pp. 466-473, 1964.

[17] - Nonlinear Systems—The Parameter Analysis and Design. New York: Wiley, 1969.

[18] J. J. E. Slotine and W. Li, Applied Nonlinear Control. Englewood Cliffs, NJ: Prentice-Hall, 1991.

[19] X. Zeng and M. G. Singh, "Approximation theory of fuzzy systemsSISO case,” IEEE Trans. Fuzzy Syst., vol. 2, pp. 162-176, Feb. 1994.

[20] - "Approximation theory of fuzzy systems-MIMO case," IEEE Trans. Fuzzy Syst., vol. 3, pp. 219-235, May 1995. 